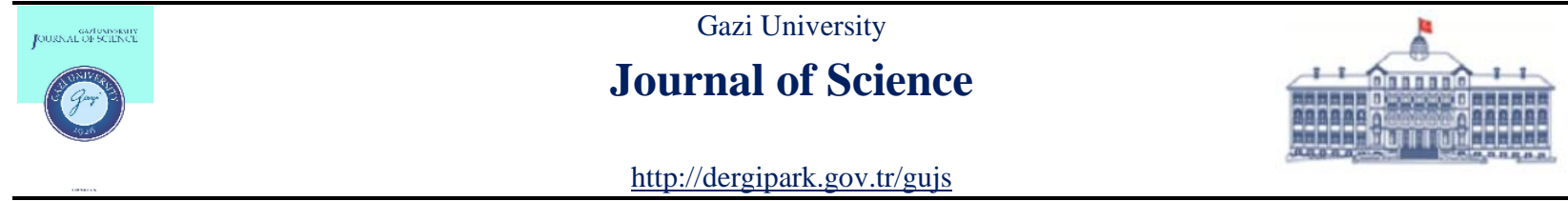

\title{
Estimation in Step-Stress Partially Accelerated Life Tests for the Power Lindley Distribution Under Progressive Censoring
}

\author{
Cagatay CETINKAYA* \\ Bingöl University, Faculty of Economics \& Administrative Sciences, Department of Business Administration, Bingöl, 12000, Turkey
}

Highlights

- This paper focuses on a step-stress accelerated life test for the power Lindley distribution.

- Progressive type-II censoring scheme is considered.

- Maximum likelihood estimation method is used with approximate confidence intervals.

\section{Article Info}

Received: 30 Jan 2020

Accepted: 10 Sep 2020

\section{Keywords}

Accelerated life test

Progressive censoring

Power lindley

Distribution

Maximum likelihood

\begin{abstract}
In this study, inference for the power Lindley distribution under a step-stress partially accelerated life test based on progressive Type-II censoring scheme is studied. The maximum likelihood estimates of the parameters and acceleration factor is investigated with their corresponding approximate confidence intervals by using asymptotic theory. The performances of the estimators and their corresponding approximate confidence intervals are evaluated with simulation studies. A real data set is used to illustrate the estimation procedure.
\end{abstract}

\section{INTRODUCTION}

In statistical reliability literature, accelerated life test (ALT) and partially accelerated life test (PALT) studies have been popular due to these tests let the experimenters control the higher stress levels to be used for components or units in the life tests. It is known that it is not easy to observe lifetimes of highly reliable components since very long lifetimes or few failures occur in limited testing time under normal operation conditions. In these cases, ALT or PALT is mostly used tests to provide early failures on components. In ALT, all test components are exposed to higher than usual stress level. On the other hand, only some of them are run under higher stress level in PALT (see [1] and [2] for details). The stress loadings in these tests can be observed in two cases as step-stress or constant stress.

The step-stress allows the experiments to choose multiple stress factors such as temperature, pressure, voltage, wind power, shock, vibration etc. in a life testing. In the step-stress partially accelerated life test (SSPALT) defined by DeGroot and Goel, 1979 [2] a life test starts with normal using conditions, if it does not fail on a pre-specified time $\tau$ it is run under an acceleration factor $\xi$ until the test terminates. By assuming $\mathrm{X}$ denote the random variable on standard conditions and $\mathrm{Y}$ denote the total lifetime of a test unit, this SSPALT model is defined as in the following relation [2]

$$
Y=f(x)=\left\{\begin{array}{cc}
X, & x \leq \tau \\
\tau+\frac{X-\tau}{\xi}, & x>\tau
\end{array}\right.
$$


where $\tau$ is the stress change time, and $\xi$ is the acceleration factor, where $\xi>1$.

On the other hand, another important topic in reliability theory is data censoring schemes (CS). It is known that the lifetimes of components or units may not be always recorded exactly. In most cases, components or units are lost or removed from the experiments before they failed and censored datasets are observed. In literature, there are many different CSs such as Type-I censoring, Type-II censoring, hybrid censoring which is a mixture of Type-I and Type II and introduced by Epstein, 1954 [3], progressive CSs which let the experimenters to remove live units on failure times.

Many reliability models are considered under different CSs. In specific of ALTs, there are various studies in the literature. For example; Meeker, 1984 [4] compared ALT plans for Weibull and lognormal models and Type-I CS, Bai et al., 1993 [5] studied lognormal distribution under Type-I censoring, Ismail, 2014 [6] studied Weibull distribution under adaptive Type-II progressively hybrid censoring, EL-Sagheer et al., 2019 [7] studied the Weibull-exponential model under progressive type-II CS, Zhao et al., 2014 [8] studied Type-I progressively hybrid censored Burr-XII data, Balakrishnan and Xie, 2000 [9] studied Type-I hybrid censored exponential model.

However, a SSPALT model under any CSs has not been considered for power Lindley (PL) distribution which is proposed by Ghitany et al., 2013 [10] as an extension of the Lindley distribution and its density and survival functions of are given by

$$
f(x ; \alpha, \beta)=\frac{\alpha \beta^{2}}{\beta+1} x^{\alpha-1}\left(1+x^{\alpha}\right) e^{-\beta x^{\alpha}}, \quad x>0, \quad \alpha, \beta>0
$$

and

$$
S(x ; \alpha, \beta)=\left(1+\frac{\beta}{\beta+1} x^{\alpha}\right) e^{-\beta x^{\alpha}}
$$

where $\alpha$ is the shape and $\beta$ is the scale parameter. Ghitany et al., 2013 [10] proved that the power Lindley distribution provides more flexibility than the Lindley distribution in terms of the shape of the density and hazard rate functions beside with its skewness and kurtosis. So, the power Lindley distribution has many advantages for modelling lifetime datasets in terms of this flexibility. Many authors focused on the power Lindley distribution in their studies. Following inference study of parameters of the PLD by Ghitany et al., 2013 [10], Valiollahi et al., 2018 [11] considered the estimation and prediction of the PL under progressively type II right censoring scheme. The PLD is also subjected to reliability studies such as stress-strength reliability by Ghitany et al., 2015 [12], Joukar et al., 2020 [13]. In terms of ALT or PALT plans, the PLD has never been studied, before. In this study, we considered the power Lindley distribution under the step-stress partially accelerated life test which is proposed by DeGroot and Goel, 1979 [2] under progressive Type-II censoring scheme with given assumptions in the following

- The experiment starts with $n$ independent and identical units which have the $P L(\alpha, \beta)$.

- All $n$ units run under normal condition until a pre-specified $\tau$ time. If it does not fail before $\tau$ time, then it is run at accelerated condition with acceleration factor $(\xi)$,

- The test is terminated with the prefixed $m$ th failure $(m \leq n)$.

- When the first failure occurs $R_{1}$ live units are randomly removed from the experiment. At the second failure, $R_{2}$ live units are randomly removed from the experiment. This test terminates with $m$ th failure and the remaining surviving units $R_{m}=n-m-\sum_{i=1}^{m-1} R_{i}$ are all removed from the experiment (Figure 1)

where $R=\left(R_{1}, R_{2}, \cdots, R_{m}\right), \sum_{i=1}^{m} R_{i}=n-m$ and $n_{u}$ is the number of failed units at normal use condition. 


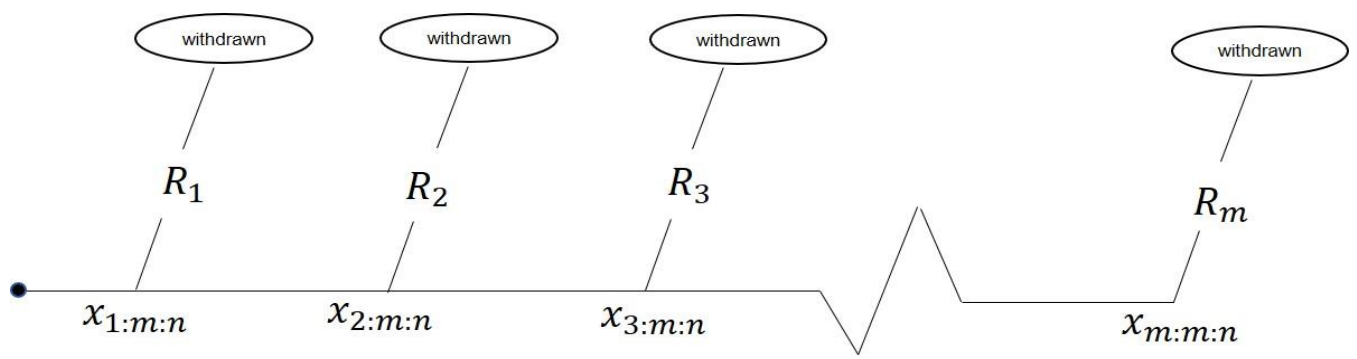

Figure 1. Structure of a progressive type-II censoring scheme

The aim of this study is making inferences for progressive Type-II censored data under SSPALT by considering the $P L(\alpha, \beta)$ distribution. Further, we aimed to investigate the effects of acceleration on the distribution. The maximum likelihood estimates (MLE) of the parameters and acceleration factor is investigated with their corresponding confidence intervals. All theoretical studies are exemplified with simulation studies and a real data example. In Section 2, we described the model and obtained the maximum likelihood estimates of the parameters. Then, approximate confidence intervals by using the asymptotic theory are given in subsection 2.1. To illustrate the theoretical outcomes, we considered some simulation studies and a real data example in Section 3.

\section{DESCRIPTION OF THE MODEL AND THE MAXIMUM LIKELIHOOD INFERENCE}

The probability density function (pdf) of the PL distribution under SSPALT, denoted by $P L(\alpha, \beta, \xi)$ can be obtained with the transformation-variable technique by using Equation (2) with (1) as follows

$$
f_{\text {SSPALT }}(x ; \alpha, \beta, \xi)= \begin{cases}\frac{\alpha \beta^{2}}{\beta+1} x^{\alpha-1}\left(1+x^{\alpha}\right) e^{-\beta x^{\alpha}}, & x \leq \tau \\ \frac{\xi \alpha \beta^{2}}{\beta+1} \gamma^{\alpha-1}\left(1+\gamma^{\alpha}\right) e^{-\beta \gamma^{\alpha}}, & x>\tau\end{cases}
$$

where $\gamma=\xi(x-\tau)+\tau$. And survival function can be obtained similary as

$$
S_{S S P A L T}(x ; \alpha, \beta, \xi)= \begin{cases}\left(1+\frac{\beta}{\beta+1} x^{\alpha}\right) e^{-\beta x^{\alpha}}, & x \leq \tau \\ \left(1+\frac{\beta}{\beta+1} \gamma^{\alpha}\right) e^{-\beta \gamma^{\alpha}}, & x>\tau .\end{cases}
$$

Here, each side of the pdf and survival function can be denoted by $f_{1}, f_{2}, S_{1}$ and $S_{2}$, respectively. Then, the MLEs of the unknown parameters and the acceleration factor, $\hat{\theta}=(\hat{\alpha}, \hat{\beta}, \hat{\xi})$, are investigated based on the proposed model above.

Firstly, the likelihood function of the progressive Type-II censored data from the PL distribution under a SSPALT can be written as in the following (see [14])

$$
L(\alpha, \beta, \xi)=c \prod_{i=1}^{n_{u}}\left[f _ { 1 } ( x _ { i } ) ( S _ { 1 } ( x _ { i } ) ^ { R _ { i } } ] \prod _ { i = n _ { u } + 1 } ^ { m } \left[f_{2}\left(x_{i}\right)\left(S_{2}\left(x_{i}\right)^{R_{i}}\right]\right.\right.
$$

where $c=n\left(n-1-R_{1}\right)\left(n-1-R_{1}-R_{2}\right) \cdots\left(n-m+1-\sum_{i=1}^{m-1} R_{i}\right)$ for 


$$
x_{1: m: n}^{R}<\cdots<x_{n_{u}: m: n}^{R}<\tau<x_{n_{u}+1: m: n}^{R}<\cdots<x_{m: m: n}^{R} .
$$

Thus, the likelihood function can be obtained by using Equations (4) and (5) in Equation (6) as follows

$$
\begin{aligned}
L(\alpha, \beta, \xi) & =c \frac{\alpha^{m} \beta^{2 m}}{(\beta+1)^{m}} \xi^{m-n_{u}} \prod_{i=1}^{n_{u}} x_{i}^{\alpha-1}\left(1+x_{i}^{\alpha}\right) e^{-\beta\left(R_{i}+1\right) x_{i}^{\alpha}}\left(1+\frac{\beta}{\beta+1} x_{i}^{\alpha}\right)^{R_{i}} \\
& \times \prod_{i=n_{u}+1}^{m} \gamma_{i}^{\alpha-1}\left(1+\gamma_{i}^{\alpha}\right) e^{-\beta\left(R_{i}+1\right) \gamma_{i}^{\alpha}}\left(1+\frac{\beta}{\beta+1} \gamma_{i}^{\alpha}\right)^{R_{i}}
\end{aligned}
$$

and the log-likelihood function is obtained as

$$
\begin{aligned}
l(\alpha, \beta, \xi) & \propto m(\ln (\alpha)+2 \ln (\beta)-\ln (\beta+1))+\left(m-n_{u}\right) \ln (\xi)+\sum_{i=1}^{n_{u}} \ln \left(1+x_{i}^{\alpha}\right) \\
& +\sum_{i=n_{u}+1}^{m} \ln \left(1+\gamma_{i}^{\alpha}\right)+(\alpha-1)\left[\sum_{i=1}^{n_{u}} \ln \left(x_{i}\right)+\sum_{i=n_{u}+1}^{m} \ln \left(\gamma_{i}\right)\right]+\sum_{i=1}^{n_{u}} R_{i} \ln \left(1+\frac{\beta}{\beta+1} x_{i}^{\alpha}\right) \\
& +\sum_{i=n_{u}+1}^{m} R_{i} \ln \left(1+\frac{\beta}{\beta+1} \gamma_{i}^{\alpha}\right)-\beta\left[\sum_{i=1}^{n_{u}}\left(R_{i}+1\right) x_{i}^{\alpha}+\sum_{i=n_{u}+1}^{m}\left(R_{i}+1\right) \gamma_{i}^{\alpha}\right] .
\end{aligned}
$$

To obtain the MLEs of the parameters, denoted by $\hat{\alpha}, \hat{\beta}$ and $\hat{\xi}$, we should equate the partial derivates of $l(\alpha, \beta, \xi)$ to zero with respect to $\alpha, \beta$ and $\xi$ respectively as given in the following

$$
\begin{aligned}
\frac{\partial l}{\partial \alpha}= & \frac{m}{\alpha}+\sum_{i=1}^{n_{u}} \ln \left(x_{i}\right)\left(1-\beta\left(1+R_{i}\right) x_{i}^{\alpha}+\frac{x_{i}^{\alpha}}{1+x_{i}^{\alpha}}\right)+\sum_{i=n_{u}+1}^{m} \ln \left(\gamma_{i}\right)\left(1-\beta\left(1+R_{i}\right) \gamma_{i}^{\alpha}+\frac{\gamma_{i}^{\alpha}}{1+\gamma_{i}^{\alpha}}\right) \\
& +\sum_{i=1}^{n_{u}} \frac{R_{i} x_{i}^{\alpha} \ln \left(x_{i}\right)}{1+x_{i}^{\alpha}+1 / \beta}+\sum_{i=1}^{n_{u}} \frac{R_{i} \gamma_{i}^{\alpha} \ln \left(\gamma_{i}\right)}{1+\gamma_{i}^{\alpha}+1 / \beta}=0 \\
\frac{\partial l}{\partial \beta}= & \frac{m(\beta+2)}{\beta(\beta+1)}-\sum_{i=1}^{n_{u}} x_{i}^{\alpha}\left(1+R_{i}\right)-\sum_{i=n_{u}+1}^{m} \gamma_{i}^{\alpha}\left(1+R_{i}\right) \\
& +\frac{1}{(\beta+1)}\left[\sum_{i=1}^{n_{u}} \frac{R_{i} x_{i}^{\alpha}}{\beta\left(1+x_{i}^{\alpha}\right)+1}+\sum_{i=n_{u}+1}^{m} \frac{R_{i} \gamma_{i}^{\alpha}}{\beta\left(1+\gamma_{i}^{\alpha}\right)+1}\right]=0 \\
\frac{\partial l}{\partial \xi}= & \frac{m-n_{u}}{\xi}+(\alpha-1) \sum_{i=1}^{n_{u}} \frac{x_{i}-\tau}{\gamma_{i}}+\sum_{i=n_{u}+1}^{m} \alpha\left(x_{i}-\tau\right) \gamma_{i}^{\alpha-1}\left[\frac{1}{1+\gamma_{i}^{\alpha}}-\beta\left(R_{i}+1\right)+\frac{R_{i}}{1+\gamma_{i}^{\alpha}+\frac{1}{\beta}}\right]=0 .
\end{aligned}
$$

However, these non-linear equations cannot be solved analytically and iterative methods such as NewtonRaphson method is needed. Thus, approximate solutions of the system of these non-linear equations be the MLEs of the parameters. 


\subsection{Approximate Confidence Intervals}

This section considers asymptotic confidence intervals for the MLEs of the parameters $\alpha, \beta$ and $\xi$. In this purpose, we firstly need the inverse of the asymptotic Fisher information matrix $I_{i, j}(\theta)=$ $E\left[-\partial^{2} l / \partial \theta_{i} \partial \theta_{j}\right]$. However, exact expressions for these expectations are very difficult to obtain in our problem. Therefore, the observed Fisher information matrix is given by $I_{i, j}(\theta)=-\partial^{2} l / \partial \theta_{i} \partial \theta_{j}$ is used as given in the following

$$
I^{-1}=\left(\begin{array}{ccc}
-\frac{\partial^{2} l}{\partial \alpha^{2}} & \frac{\partial^{2} l}{\partial \alpha \partial \beta} & -\frac{\partial^{2} l}{\partial \alpha \partial \xi} \\
-\frac{\partial^{2} l}{\partial \beta \partial \alpha} & -\frac{\partial^{2} l}{\partial \beta^{2}} & -\frac{\partial^{2} l}{\partial \beta \partial \xi} \\
-\frac{\partial^{2} l}{\partial \xi \partial \alpha} & -\frac{\partial^{2} l}{\partial \xi \partial \beta} & -\frac{\partial^{2} l}{\partial \xi^{2}}
\end{array}\right)^{-1}=\left(\begin{array}{lll}
I_{11} & I_{12} & I_{13} \\
I_{21} & I_{22} & I_{23} \\
I_{31} & I_{32} & I_{33}
\end{array}\right)
$$

and this inverse asymptotic Fisher information matrix is equal to

$$
I^{-1}=\left(\begin{array}{ccc}
\operatorname{Var}(\hat{\alpha}) & \operatorname{Cov}(\hat{\alpha}, \hat{\beta}) & \operatorname{Cov}(\hat{\alpha}, \hat{\xi}) \\
\operatorname{Cov}(\hat{\beta}, \hat{\alpha}) & \operatorname{Var}(\hat{\beta}) & \operatorname{Cov}(\hat{\beta}, \hat{\xi}) \\
\operatorname{Cov}(\hat{\xi}, \hat{\alpha}) & \operatorname{Cov}(\hat{\xi}, \hat{\beta}) & \operatorname{Var}(\hat{\xi})
\end{array}\right)
$$

Thus, asymptotic variances of the parameters can be obtained as

$$
\begin{aligned}
& \operatorname{Var}(\hat{\alpha})=\left(I_{33} I_{22}-I_{23}^{2}\right) / \operatorname{det}(I) \\
& \operatorname{Var}(\hat{\beta})=\left(I_{33} I_{11}-I_{13}^{2}\right) / \operatorname{det}(I) \\
& \operatorname{Var}(\hat{\xi})=\left(I_{22} I_{11}-I_{12}^{2}\right) / \operatorname{det}(I)
\end{aligned}
$$

where $\operatorname{det}(I)$ is the determinant of the Fisher information matrix and obtained as in the following

$$
\operatorname{det}(I)=I_{11}\left(I_{33} I_{22}-I_{23}^{2}\right)-I_{12}\left(I_{33} I_{12}-I_{23} I_{13}\right)+I_{13}\left(I_{23} I_{12}-I_{22} I_{13}\right) .
$$

Then, the $100(1-\delta) \%$ asymptotic confidence intervals of $\hat{\alpha}, \hat{\beta}$ and $\hat{\xi}$ can be constructed by

$$
\hat{\alpha} \mp \mathrm{z}_{\delta / 2} \sqrt{\operatorname{Var}(\hat{\alpha})} ; \quad \hat{\beta} \mp \mathrm{z}_{\delta / 2} \sqrt{\operatorname{Var}(\hat{\beta})} ; \hat{\xi} \mp \mathrm{z}_{\delta / 2} \sqrt{\operatorname{Var}(\hat{\xi})}
$$

where $\mathrm{z}_{\delta}$ is $100 \delta$ th percentile of standard normal distribution $N(0,1)$. 
Here, elements of the Fisher information matrix are the second partial derivatives of the log-likelihood function (8) with respect to $\alpha, \beta$ and $\xi$ respectively and are obtained as follows

$$
\begin{aligned}
& \frac{\partial^{2} l}{\partial \alpha^{2}}=-\frac{m}{\alpha^{2}}+\sum_{i=1}^{n_{u}} x_{i}^{\alpha}\left(\ln x_{i}\right)^{2}\left[\frac{1}{\left(1+x_{i}^{\alpha}\right)^{2}}-\left(1+R_{i}\right) \beta-\frac{R_{i}(1+1 / \beta)}{\left(1+x_{i}^{\alpha}+1 / \beta\right)^{2}}\right] \\
& +\sum_{i=n_{u}+1}^{m} \gamma_{i}^{\alpha}\left(\ln \gamma_{i}\right)^{2}\left[\frac{1}{\left(1+\gamma_{i}^{\alpha}\right)^{2}}-\left(1+R_{i}\right) \beta-\frac{R_{i}(1+1 / \beta)}{\left(1+\gamma_{i}^{\alpha}+1 / \beta\right)^{2}}\right] \\
& \frac{\partial^{2} l}{\partial \alpha \partial \beta}=\sum_{i=1}^{n_{u}} \frac{R_{i} x_{i}^{\alpha} \ln \left(x_{i}\right)}{\beta^{2}\left(1+x_{i}^{\alpha}+1 / \beta\right)^{2}}+\sum_{i=1}^{n_{u}} \frac{R_{i} \gamma_{i}^{\alpha} \ln \left(\gamma_{i}\right)}{\beta^{2}\left(1+\gamma_{i}^{\alpha}+1 / \beta\right)^{2}}-\sum_{i=1}^{n_{u}}\left(1+R_{i}\right) x_{i}^{\alpha} \ln \left(x_{i}\right) \\
& -\sum_{i=n_{u}+1}^{m}\left(1+R_{i}\right) \gamma_{i}^{\alpha} \ln \left(\gamma_{i}\right) \\
& \frac{\partial^{2} l}{\partial \alpha \partial \xi}=\sum_{i=n_{u}+1}^{m}\left[\frac{x_{i}-\tau}{\gamma_{i}}\left(\frac{\gamma_{i}^{\alpha}}{1+\gamma_{i}^{\alpha}}+1-\beta \gamma_{i}^{\alpha}\left(1+R_{i}\right)\right)+\log \left(\gamma_{i}\right)\left(\frac{\alpha\left(x_{i}-\tau\right) \gamma_{i}^{\alpha-1}}{\left(1+\gamma_{i}^{\alpha}\right)^{2}}\right)\right. \\
& \left.-\alpha \beta\left(x_{i}-\tau\right)\left(1+R_{i}\right) \gamma_{i}^{\alpha-1}+\frac{\alpha R_{i}\left(x_{i}-\tau\right) \gamma_{i}^{\alpha-1}(1+1 / \beta)}{\left(1+\gamma_{i}^{\alpha}+1 / \beta\right)^{2}}\right] \\
& \frac{\partial^{2} l}{\partial \beta^{2}}=-\frac{m\left(\beta^{2}+4 \beta+2\right)}{\beta^{2}(\beta+1)^{2}} \\
& -\frac{1}{(1+\beta)^{2}}\left[\sum_{i=1}^{n_{u}} \frac{R_{i} x_{i}^{\alpha}\left[1+\left(1+x_{i}^{\alpha}\right)(1+2 \beta)\right]}{\left(1+\beta\left(1+x_{i}^{\alpha}\right)\right)^{2}}-\sum_{i=n_{u}+1}^{m} \frac{R_{i} \gamma_{i}^{\alpha}\left[1+\left(1+\gamma_{i}^{\alpha}\right)(1+2 \beta)\right]}{\left(1+\beta\left(1+\gamma_{i}^{\alpha}\right)\right)^{2}}\right] \\
& \frac{\partial^{2} l}{\partial \beta \partial \xi}=\sum_{i=n_{u}+1}^{m} \frac{\alpha R_{i}\left(x_{i}-\tau\right) \gamma_{i}^{\alpha-1}}{\left[1+\beta\left(1+\gamma_{i}^{\alpha}\right)\right]^{2}}-\sum_{i=n_{u}+1}^{m} \alpha\left(x_{i}-\tau\right)\left(1+R_{i}\right) \gamma_{i}^{\alpha-1} \\
& \frac{\partial^{2} l}{\partial \xi^{2}}=-\frac{m-n_{u}}{\xi^{2}}+(\alpha-1) \sum_{i=n_{u}+1}^{m}\left(\frac{x_{i}-\tau}{\gamma_{i}}\right)^{2}-\alpha(\alpha-1) \beta \sum_{i=n_{u}+1}^{m}\left(1+R_{i}\right)\left(x_{i}-\tau\right)^{2} \gamma_{i}^{\alpha-2} \\
& +\sum_{i=n_{u}+1}^{m} \alpha\left(x_{i}-\tau\right)^{2} \gamma_{i}^{\alpha-2}\left[\frac{\alpha-1-\gamma_{i}^{\alpha}}{\left(1+\gamma_{i}^{\alpha}\right)^{2}}+\frac{R_{i}\left[(\alpha-1)(1+1 / \beta)-\gamma_{i}^{\alpha}\right]}{\left(1+\gamma_{i}^{\alpha}+1 / \beta\right)^{2}}\right] \text {. }
\end{aligned}
$$

\section{SIMULATION STUDIES}

In this section, performances of the estimation procedures are evaluated with some simulation studies. The ML estimates of the parameters are evaluated in terms of their biases and mean squared errors (MSE) for different choices of parameters and sample sizes based on four different censoring schemes (CS) which is given in the following. In addition, corresponding approximate confidence intervals are obtained and reported in terms of their average lengths (AL) and coverage probabilities $(\mathrm{CP})$.

CS-A: $R_{1}=n-m$ and $R_{i}=0$ for $i=2, \cdots, m$. 
CS-B: $R_{m}=n-m$ and $R_{i}=0$ for $i=1, \cdots, m-1$.

CS-C: $R_{i}=1$ for $i=1, \cdots, n-m$ and $R_{i}=0$ for $i=n-m+1, \cdots, m$.

CS-D: $R_{i}=1$ for $i=2 m-n+1, \cdots, m$ and $R_{i}=0$ for $i=1, \cdots, 2 m-n$.

For this purpose, by using the algorithm which is defined by Balakrishnan and Sandhu, 1995 [15] and given in below, we firstly generate random samples from the PL distribution with parameters $(\alpha=1.5, \beta=$ $0.5, \xi=1.2)$ at stress change times $\tau=1.5$ and $\tau=2$, respectively.

Step 1: Generate $\mathrm{m}$ independent $W_{1}, W_{2}, \cdots, W_{m}$ observations from $U(0,1)$.

Step 2: Set $V_{i}=W_{i}^{1 /\left(1+R_{m}+R_{m-1}+\cdots+R_{m-i+1}\right)}$ for $i=1,2, \cdots, m$.

Step 3: Set $U_{i}=1-V_{m} V_{m-1} \cdots V_{m-i+1}$ for $i=1,2, \cdots, m$ as the required progressive Type-II censored sample from the $U(0,1)$.

Step 4: Set $X_{i}=F^{-1}\left(U_{i}\right)$ for $i=1,2, \cdots, m$ where $F^{-1}\left(U_{i}\right)$ is the inverse cdf of the distribution which is given in Equation (9).

Then, $X_{1}, X_{2}, \cdots, X_{m}$ is the required progressive Type-II censored sample from the power Lindley distribution under SSPALT.

To obtain such a random sample, the quantile function of the power Lindley distribution under SSPALT is needed. Recently, Jodra, 2010 [16] obtained the quantile function of Lindley distribution and Ghitany et al, 2013 [13] expanded this for power Lindley distribution. Following to these results, we obtained the quantile function of the power Lindley distribution under SSPALT by using equation (5) as

$$
F_{A L T}^{-1}(x)=f(x)= \begin{cases}{\left[-1-\frac{1}{\beta}-\frac{1}{\beta} W_{-1}\left(-\frac{\beta+1}{e^{\beta+1}}(1-u)\right)\right]^{1 / \alpha},} & x \leq \tau \\ \frac{1}{\xi}\left[-1-\frac{1}{\beta}-\frac{1}{\beta} W_{-1}\left(-\frac{\beta+1}{e^{\beta+1}}(1-u)\right)\right]^{1 / \alpha}-\frac{\tau}{\xi}+\tau, & x>\tau\end{cases}
$$

where $W_{-1}($.$) denotes the negative branch of the Lambert W$ function.

Under the given progressive censoring schemes for various sample sizes $(n ; m)$ such as $(25 ; 20),(30 ; 25)$, $(40 ; 30),(50 ; 40)$, we used 1000 simulated samples and the MLEs with $95 \%$ CIs for the parameters $\alpha, \beta$ and $\xi$ are obtained and reported in Table 1 for $\tau=1.5$ and in Table 2 for $\tau=2$.

We observe that, for the shape parameter $\alpha$, the bias and MSE values decrease as the sample size increases, in all cases, as expected. On the other hand, biases and MSEs of the scale parameter and acceleration factor, $\beta$ and $\xi$, have almost equal and close to zero values in all cases. Average lengths of the credible intervals are decreasing in parallel to increasing sample sizes in both cases. It is noticed that credible interval for $\xi$ does not perform well. They have larger lengths and unexpected CPs. The main reason of this result is the values of $\xi$ which maximizing the log-likelihood function. In Section 2, we mentioned that iterative methods such as Newton-Raphson method is needed for MLEs. In these methods, restriction on $\xi$, that is $\xi>1$, influence the optimizing values. Therefore, variances of $\xi$ give large values in all cases. This situation can explain the unexpected results on their credible intervals. According to the differences between censoring schemes, we observed that removing items at last of the experiments (CS-B and CS-D) cause increasing on biases and MSEs of the shape parameter $\alpha$. On contrast, there are decreasing on $\beta$ and $\xi$, in the same cases. These results are related to effects of the acceleration factor on values. In parallel to increasing in stress change time $\tau$, average confidence lengths in all cases. 
On the other hand, we tried many different combinations of the values for the parameters but not reported here all of them. It is clear that, selection of stress change time $\tau$, acceleration factor $\xi$ and censoring schemes are very important to obtain consistent results. They must be selected to the structure of the dataset and expectations of the experimenters. In this study, we only used some combinations of parameters to illustrate our theoretical findings.

Table 1. The biases and MSEs of the MLEs for the parameters at $\alpha=2, \beta=0.5$ and $\xi=1.2$ at $\tau=1.5$ with corresponding ACIs. First rows of each sample report the biases and the average lengths. Second rows of each sample report the MSEs and the CPs of the intervals

\begin{tabular}{|c|c|c|c|c|c|c|c|}
\hline \multirow[b]{2}{*}{$(n, m)$} & \multirow[b]{2}{*}{$C S$} & \multicolumn{3}{|c|}{ MLEs } & \multicolumn{3}{|c|}{ ACIs } \\
\hline & & $\alpha$ & $\beta$ & $\xi$ & $\alpha$ & $\beta$ & $\xi$ \\
\hline \multirow[t]{8}{*}{$(25,20)$} & I & 0.15289 & 0.02857 & 0.08294 & 2.74975 & 0.55255 & 4.59497 \\
\hline & & 0.02337 & 0.00082 & 0.00688 & 97.81 & 96.41 & 100 \\
\hline & II & 0.23760 & 0.01689 & 0.03902 & 2.71059 & 0.51893 & 3.89165 \\
\hline & & 0.05645 & 0.00029 & 0.00152 & 98.28 & 96.68 & 100 \\
\hline & III & 0.16185 & 0.02649 & 0.05495 & 2.71823 & 0.53284 & 4.40790 \\
\hline & & 0.02620 & 0.00070 & 0.00302 & 97.87 & 96.78 & 100 \\
\hline & IV & 0.22844 & 0.01859 & 0.03857 & 2.73339 & 0.52028 & 3.94210 \\
\hline & & 0.05219 & 0.00035 & 0.00149 & 98.29 & 96.75 & 100 \\
\hline \multirow[t]{8}{*}{$(30,20)$} & I & 0.14606 & 0.03070 & 0.07676 & 2.58722 & 0.53748 & 4.38261 \\
\hline & & 0.02133 & 0.00094 & 0.00589 & 97.83 & 96.62 & 100 \\
\hline & II & 0.27798 & 0.00644 & 0.09815 & 2.38715 & 0.46821 & 3.81606 \\
\hline & & 0.07727 & 0.00004 & 0.00963 & 98.08 & 96.18 & 100 \\
\hline & III & 0.16709 & 0.02414 & 0.04377 & 2.59683 & 0.49271 & 4.16604 \\
\hline & & 0.02792 & 0.00058 & 0.00192 & 98.56 & 96.94 & 100 \\
\hline & IV & 0.25406 & 0.01703 & 0.05593 & 2.49161 & 0.47800 & 3.72025 \\
\hline & & 0.06455 & 0.00029 & 0.00313 & 98.25 & 96.31 & 100 \\
\hline \multirow[t]{8}{*}{$(40,30)$} & I & 0.13926 & 0.03062 & -0.00954 & 2.32167 & 0.44764 & 3.49432 \\
\hline & & 0.01939 & 0.00094 & 0.00009 & 98.39 & 96.08 & 100 \\
\hline & II & 0.23829 & 0.01810 & -0.04156 & 2.01051 & 0.40431 & 2.67072 \\
\hline & & 0.05678 & 0.00033 & 0.00173 & 98.77 & 95.86 & 100 \\
\hline & III & 0.14596 & 0.02674 & -0.01191 & 2.21106 & 0.41907 & 3.32889 \\
\hline & & 0.02130 & 0.00071 & 0.00014 & 98.75 & 96.14 & 100 \\
\hline & IV & 0.22235 & 0.02099 & -0.04292 & 2.08883 & 0.40742 & 2.81148 \\
\hline & & 0.04944 & 0.00044 & 0.00184 & 98.88 & 95.84 & 100 \\
\hline \multirow[t]{8}{*}{$(50,40)$} & I & 0.13545 & 0.03380 & -0.06240 & 2.01099 & 0.39115 & 2.83617 \\
\hline & & 0.01835 & 0.00114 & 0.00389 & 98.98 & 96.08 & 100 \\
\hline & II & 0.20436 & 0.02352 & -0.08133 & 1.79959 & 0.36171 & 2.32518 \\
\hline & & 0.04176 & 0.00055 & 0.00661 & 99.18 & 95.96 & 100 \\
\hline & III & 0.13554 & 0.03155 & -0.05853 & 1.91283 & 0.37072 & 2.76655 \\
\hline & & 0.01837 & 0.00100 & 0.00343 & 99.14 & 95.87 & 100 \\
\hline & IV & 0.19484 & 0.02551 & -0.08076 & 1.82849 & 0.36210 & 2.39726 \\
\hline & & 0.03796 & 0.00065 & 0.00652 & 99.20 & 95.89 & 100 \\
\hline
\end{tabular}


Table 2. The biases and MSEs of the MLEs for the parameters at $\alpha=2, \beta=0.5$ and $\xi=1.2$ at $\tau=2$ with corresponding ACIs. First rows of each sample report the biases and the average lengths. Second rows of each sample report the MSEs and the CPS of the intervals

\begin{tabular}{|c|c|c|c|c|c|c|c|}
\hline \multirow[b]{2}{*}{$(n, m)$} & \multirow[b]{2}{*}{$C S$} & \multicolumn{3}{|c|}{ MLES } & \multicolumn{3}{|c|}{$A C I s$} \\
\hline & & $\alpha$ & $\beta$ & $\xi$ & $\alpha$ & $\beta$ & $\xi$ \\
\hline \multirow[t]{8}{*}{$(25,20)$} & I & 0.19464 & 0.00594 & 0.07026 & 2.02403 & 0.56230 & 3.50154 \\
\hline & & 0.03788 & 0.00004 & 0.00494 & 98.39 & 95.98 & 100 \\
\hline & II & 0.18308 & -0.03926 & 0.33056 & 1.75556 & 0.49685 & 4.65225 \\
\hline & & 0.03352 & 0.00154 & 0.10927 & 95.83 & 93.21 & 100 \\
\hline & III & 0.19305 & 0.00593 & 0.07867 & 1.95915 & 0.53811 & 3.50847 \\
\hline & & 0.03727 & 0.00004 & 0.00619 & 98.30 & 95.85 & 100 \\
\hline & IV & 0.24300 & -0.01612 & 0.85578 & 1.81269 & 0.50715 & 6.54750 \\
\hline & & 0.05905 & 0.00026 & 0.73236 & 96.06 & 93.39 & 100 \\
\hline \multirow[t]{8}{*}{$(30,20)$} & I & 0.18372 & 0.00934 & 0.07707 & 1.94142 & 0.54564 & 3.46656 \\
\hline & & 0.03375 & 0.00009 & 0.00594 & 98.21 & 95.88 & $\begin{array}{c}100 \\
16.0436\end{array}$ \\
\hline & II & -0.39966 & -0.21370 & 1.53358 & 1.54004 & 0.43493 & 5 \\
\hline & & 0.15973 & 0.04567 & 2.35186 & 94.27 & 89.48 & 100 \\
\hline & III & 0.18462 & 0.00559 & 0.10725 & 1.86478 & 0.49085 & 3.57094 \\
\hline & & 0.03409 & 0.00003 & 0.01150 & 97.73 & 95.17 & 100 \\
\hline & IV & 0.18565 & -0.02797 & 0.67442 & 1.61391 & 0.45282 & 5.80913 \\
\hline & & 0.03447 & 0.00078 & 0.45484 & 95.49 & 93.07 & 100 \\
\hline \multirow[t]{8}{*}{$(40,30)$} & I & 0.17958 & 0.00679 & -0.01157 & 1.63667 & 0.45930 & 2.63884 \\
\hline & & 0.03225 & 0.00005 & 0.00013 & 98.59 & 96.17 & 100 \\
\hline & II & 0.16054 & -0.04534 & 0.27898 & 1.32828 & 0.38879 & 4.03072 \\
\hline & & 0.02577 & 0.00206 & 0.07783 & 92.65 & 92.42 & 100 \\
\hline & III & 0.17721 & 0.00631 & -0.00161 & 1.55899 & 0.42581 & 2.62870 \\
\hline & & 0.03140 & 0.00004 & 0.00001 & 98.487 & 95.52 & 100 \\
\hline & IV & 0.24219 & -0.00909 & 0.14254 & 1.37233 & 0.39713 & 3.02446 \\
\hline & & 0.05865 & 0.00008 & 0.02032 & 94.44 & 93.46 & 100 \\
\hline \multirow[t]{8}{*}{$(50,40)$} & I & 0.17283 & 0.00814 & -0.06199 & 1.39885 & 0.40243 & 2.17124 \\
\hline & & 0.02987 & 0.00007 & 0.00384 & 98.63 & 96.22 & 100 \\
\hline & II & 0.23292 & -0.00956 & 0.02889 & 1.17860 & 0.35189 & 2.39046 \\
\hline & & 0.05425 & 0.00009 & 0.00083 & 93.76 & 94.16 & 100 \\
\hline & III & 0.16989 & 0.00861 & -0.05689 & 1.33653 & 0.37811 & 2.13371 \\
\hline & & 0.02886 & 0.00007 & 0.00324 & 98.55 & 95.81 & 100 \\
\hline & IV & 0.23422 & -0.00343 & -0.00073 & 1.20720 & 0.35621 & 2.23493 \\
\hline & & 0.05486 & 0.00001 & 0.00001 & 94.84 & 94.51 & 100 \\
\hline
\end{tabular}

\subsection{Illustrative Example}

In this subsection, we considered the data of the tensile strength, measured in GPa, of 69 carbon fibers tested under tension at gauge lengths of $20 \mathrm{~mm}$ (Bader and Priest, 1982 [17]), which is fitted by power Lindley distribution by Ghitany et al., 2013 [13], before. The data set is given as 
$1.312,1.314,1.479,1.552,1.700,1.803,1.861,1.865,1.944,1.958,1.966,1.997,2.006,2.021,2.027$, $2.055,2.063,2.098,2.140,2.179,2.224,2.240,2.253,2.270,2.272,2.274,2.301,2.301,2.359,2.382$, $2.382,2.426,2.434,2.435,2.478,2.490,2.511,2.514,2.535,2.554,2.566,2.570,2.586,2.629,2.633$, $2.642,2.648,2.684,2.697,2.726,2.770,2.773,2.800,2.809,2.818,2.821,2.848,2.880,2.954,3.012$, $3.067,3.084,3.090,3.096,3.128,3.233,3.433,3.585,3.585$.

Ghitany et al., 2013 [13] fit the power Lindley distribution as well as the well-known two-parameter Gompertz, gamma and Weibull distributions and showed that power Lindley distribution provides the best fit among all the considered models.

We have generated a progressively censored sample using a random censoring scheme as $R=$ $(0,1,0,1,1,2,0,0,2,2,1,1,1,2,0,0,1,2,2,0,0,1,1,0,0,0,0,0,0,0,1,1,0,2,0,1,1,1,1,0)$ from the tensile strength data with $n=69$ and $m=40$. Then, the censoring scheme and the corresponding progressively censored sample are given in Table 3.

Table 3. Progressively censored tensile strength data

\begin{tabular}{|c|c|c|c|c|c|c|c|c|c|c|c|}
\hline$i$ & $X_{i}$ & $R_{i}$ & $i$ & $X_{i}$ & $R_{i}$ & $i$ & $X_{i}$ & $R_{i}$ & $i$ & $X_{i}$ & $R_{i}$ \\
\hline 1 & 1.312 & 0 & 11 & 2.179 & 1 & 21 & 2.554 & 0 & 31 & 2.773 & 1 \\
\hline 2 & 1.314 & 1 & 12 & 2.240 & 1 & 22 & 2.566 & 1 & 32 & 2.809 & 1 \\
\hline 3 & 1.552 & 0 & 13 & 2.270 & 1 & 23 & 2.586 & 1 & 33 & 2.821 & 0 \\
\hline 4 & 1.700 & 1 & 14 & 2.274 & 2 & 24 & 2.633 & 0 & 34 & 2.848 & 2 \\
\hline 5 & 1.861 & 1 & 15 & 2.359 & 0 & 25 & 2.642 & 0 & 35 & 3.012 & 0 \\
\hline 6 & 1.944 & 2 & 16 & 2.382 & 0 & 26 & 2.648 & 0 & 36 & 3.067 & 1 \\
\hline 7 & 1.997 & 0 & 17 & 2.382 & 1 & 27 & 2.648 & 0 & 37 & 3.090 & 1 \\
\hline 8 & 2.006 & 0 & 18 & 2.434 & 2 & 28 & 2.697 & 0 & 38 & 3.128 & 1 \\
\hline 9 & 2.021 & 2 & 19 & 2.490 & 2 & 29 & 2.726 & 0 & 39 & 3.433 & 1 \\
\hline 10 & 2.063 & 2 & 20 & 2.535 & 0 & 30 & 2.770 & 0 & 40 & 3.585 & 0 \\
\hline
\end{tabular}

We take stress change time $\tau$ as $\tau=2.75$ and $\tau=3$, respectively. For the first case, that is for $\tau=2.75$, we obtained $\hat{\alpha}=3.7848, \hat{\beta}=0.0379$ and $\hat{\xi}=1.0010$ and their corresponding approximate confidence intervals are $(2.7315,4.8381)$ with length $2.1066,(0.0008,0.0751)$ with length 0.0743 and $(0.3606,1.6414)$ with length 1.2808 , respectively. For the other $\tau=3$ value, we obtained $\hat{\alpha}=3.7496$, $\hat{\beta}=0.0390$ and $\hat{\xi}=1.0655$ and their corresponding approximate confidence intervals are $(2.8388,4.6603)$ with length $1.8215,(0.0044,0.0737)$ with length 0.0693 and $(0.2381,1.8928)$ with length 1.6547, respectively. Since the range of the data is not so large and stress change times are close to each other, we obtained similar results in our data study. Various censoring schemes and stress change times can be considered for further findings.

\section{CONCLUSIONS}

In this study, we considered the power Lindley distribution in SSPALT due to its flexibility than the Lindley distribution in terms of the shape of the density and hazard rate functions beside with its skewness and kurtosis. We studied this problem under progressive Type-II censoring scheme. ML estimations and approximate confidence intervals of the parameters are obtained. The simulation studies showed that our theoretical results are consistent and applicable. To obtain an alternative estimation procedure, we studied on Bayes estimation method, also. However, based on the different prior distributions, posterior densities of the parameters cannot be obtained clearly. To overcome this adversity, we mostly focused on MCMC methods by using Metropolis-Hasting and importance sampling methods. Unfortunately, posterior densities and their corresponding credible intervals do not perform well and unexpected results from the theoretical 
expectations. Therefore, we reported only MLE results in this study. On the other hand, the studies on PL distribution is still open problem for other ALT plans under various censoring schemes.

\section{CONFLICTS OF INTEREST}

No conflict of interest was declared by the author.

\section{ACKNOWLEDGEMENT}

The author would like to thank the anonymous reviewers for their valuable comments and suggestions which were helpful in improving the paper.

\section{REFERENCES}

[1] Nelson, W.B., “Accelerated testing: statistical models, test plans, and data analysis". John Wiley and Sons, (2009).

[2] DeGroot, M.H., Goel, P. K., "Bayesian estimation and optimal designs in partially accelerated life testing. Reliability Engineering \& System Safety, 40(1): 85-92, (1979).

[3] Epstein, B., "Truncated life tests in the exponential case". The Annals of Mathematical Statistics, 555-564, (1954).

[4] Meeker, W.Q., "A Comparison of Accelerated Life Test Plans for Weibull and Lognormal Distributions and Type-I Censoring", Technometrics, 26(2): 157-171, (1984).

[5] Bai, D.S., Chung, S.W., Chun, Y.R., "Optimal design of partially accelerated life tests for the lognormal distribution under type I censoring”, Naval Research Logistics Quarterly, 26(2): 223$235,(1993)$.

[6] Ismail, A.A., "Inference for a step-stress partially accelerated life test model with an adaptive Type-II progressively hybrid censored data from Weibull distribution", Journal of Computational and Applied Mathematics, 260: 533-542, (2014).

[7] EL-Sagheer, R.M., Mahmoud, M.A., Nagaty, H., "Inferences for Weibull-Exponential Distribution Based on Progressive Type-II Censoring Under Step-Stress Partially Accelerated Life Test Model”, Journal of Statistical Theory and Practice, 13(1): 14 (2019).

[8] Zhao, J., Shi, Y., Yan, W., "Inference for constant-stress accelerated life test with Type-I progressively hybrid censored data from Burr-XII distribution", Journal of Systems Engineering and Electronics", 25(2): 340-348, (2014).

[9] Balakrishnan, N., Xie, Q., "Exact inference for a simple step-stress model with Type-I hybrid censored data from the exponential distribution", Journal of Statistical Planning and Inference, 137(11): 268-329, (2007).

[10] Ghitany, M.E., Al-Mutairi, D.K., Balakrishnan, N., Al-Enezi, L. J., "Power Lindley distribution and associated inference", Computational Statistics \& Data Analysis, 64: 20-33, (2013).

[11] Valiollahi, R., Raqab, M.Z., Asgharzadeh, A., Alqallaf, F.A., "Estimation and prediction for power Lindley distribution under progressively type II right censored samples", Mathematics and Computers in Simulation, 149, 32-47, (2018). 
[12] Ghitany, M.E., Al-Mutairi, D.K., Aboukhamseen, S.M., "Estimation of the reliability of a stressstrength system from power Lindley distributions", Communications in Statistics-Simulation and Computation, 44(1), 118-136, (2015).

[13] Joukar, A., Ramezani, M., \& MirMostafaee, S.M.T.K., "Estimation of P (X> Y) for the power Lindley distribution based on progressively type II right censored samples", Journal of Statistical Computation and Simulation, 90(2), 355-389, (2020).

[14] Balakrishnan, N., Aggarwala, R., "Progressive censoring: theory, methods, and applications." Springer Science \& Business Media. (2000).

[15] Balakrishnan, N., Sandhu, R.A., "A simple simulational algorithm for generating progressive Type-II censored samples", The American Statistician, 49(2): 229-230, (1995).

[16] Jodra, P., "Computer generation of random variables with Lindley or Poisson-Lindley distribution via the Lambert W function", Mathematics and Computers in Simulation, 81(4): 851$859,(2010)$.

[17] Bader, M.G., Priest, A.M., "Statistical aspects of fiber and bundle strength in hybrid composites", Hayashi, T., Kawata, K., Umekawa, S. (Eds.), Progress in Science and Engineering Composites, ICCM-IV, Tokyo, pp. 1129-1136, (1982). 